\title{
Commentary on "Are Bone Allografts Safe and Effective for Today's Dental Practitioner?"
}

\section{Claudia Florina Andreescu*}

Department of Prosthetics, Faculty of Dental Medicine, University Titu Maiorescu, Bucharest, Romania

Corresponding author: Claudia Florina Andreescu, Department of Prosthetics, Faculty of Dental Medicine,

University Titu Maiorescu, Bucharest, Romania,Tel: +4021-325-1416; E-mail: claudiaandreescu@gmail.com; claudia_andreescu@yahoo.com

Rec Date: April 01, 2015; Acc Date: April 19, 2015; Pub Date: April 24, 2015

Copyright: ( 2015 Andreescu CF. This is an open-access article distributed under the terms of the Creative Commons Attribution License, which permits unrestricted use, distribution, and reproduction in any medium, provided the original author and source are credited.

\section{Commentary}

I would like address my congratulations to the authors of the article titled: “Are Bone Allografts Safe and Effective for Today's Dental Practitioner?" [1]. I believe, it is the first study assessing entirely a type of bone allograft for dental use. This study considers processing, sterility, and proven clinical performance of OraGraft, a highly disinfected, terminally sterilized bone allograft used for dental procedures that require bone regeneration.Certainly, more than a few points could be enlightened about this study. Firstly, use of bone allografts in filling periodontal, mandibular and maxillary defects is now an accepted, commonly used procedure with proven efficacy [2], but allograft safety is of great concern to all dental practitioners as well as their patients [3]. Selecting suitable bone allograft materials concerns dental practitioner with regards to their patients' safety and the clinical efficacy of the graft and as consequence occurs studies which inform dental practitioner about processing allografts [4].

The main concern about processing allograft is killing viruses. The processing of OraGraft removes blood and lipids from entire graft not only on surface graft and association with gamma irradiation kills viruses. The second point is about strength of highly processed bone allograft which depend on gamma irradiation dose as well as capacity of killing viruses. The processing implies low gamma irradiation dose at low temperature, which keep optimal residual calcium range at 10-6 Sterility Assurance Level. Numerous clinical studies demonstrate efficacy of this type bone allograft in treatment of bone defects in periodontology and dental implantology over the years. A last point, but not the leastis about osteoconductive and osteoinductive ability of allografts which generate the formation of new osteoblasts and support a faster integration. This point is enlightened by histological evaluation that demonstrate mineralized composite network of allograft granules connected by newly formed bone and osteoblast activities six months after a sinus elevation technique. There is a need for more histological studies to prove osteoinductive ability of bone allograft in association with other bone augmentation techniques.

\section{References}

1. Samsell BS, Moore M, Bertasi G, Spinato S, Bernardello, et al. (2014) Are Bone Allografts Safe and Effective for Today's Dental Practitioner? Dentistry 4: 260.

2. Grover V, Kapoor A, Malhotra R, Sachdeva S. (2011) Bone allografts: A review of safety and efficacy. Indian J Dent Res 22: 496.

3. Holzclaw D, Toscano N, Eisenlohr L, Callan D (2008) The safety of bone allografts used in dentistry. J Am Dent Assoc 139: 1192-1199.

4. Malinin TI, Temple HT, Garg AK (2014) Bone Allografts in Dentistry: A Review. Dentistry 4: 199. 